\title{
Symplectic invariants, entropic measures and correlations of Gaussian states
}

\author{
Alessio Serafini, Fabrizio Illuminati and Silvio De Siena \\ Dipartimento di Fisica "E. R. Caianiello", Università di Salerno, INFM UdR Salerno, \\ INFN Sez. Napoli, Gruppo Collegato di Salerno, Via S. Allende, 84081 Baronissi (SA), Italy
}

\begin{abstract}
We present a derivation of the Von Neumann entropy and mutual information of arbitrary two-mode Gaussian states, based on the explicit determination of the symplectic eigenvalues of a generic covariance matrix. The key role of the symplectic invariants in such a determination is pointed out. We show that the Von Neumann entropy depends on two symplectic invariants, while the purity (or the linear entropy) is determined by only one invariant, so that the two quantities provide two different hierarchies of mixed Gaussian states. A comparison between mutual information and entanglement of formation for symmetric states is considered, remarking the crucial role of the symplectic eigenvalues in qualifying and quantifying the correlations present in a generic state.
\end{abstract}

December 12, 2003

\section{Introduction}

Quantum information with continuous variable systems is rapidly developing and appears to yield very promising perspectives concerning both experimental realizations and general theoretical insights. In such a context, Gaussian states play a prominent role, both in view of their conceptual importance and for their relevance in experimental applications, and have attracted most of the attention of the researchers in the field [1]. They are the easiest states that can be created and controlled in the laboratory [2], and have been successfully exploited in quantum cryptography [3] and quantum teleportation protocols [4]. Moreover, they are possible candidates for continuous variable quantum computation processing [5] 1 ].

In particular, two-mode Gaussian states aroused great interest in later years, being the simplest prototype of a continuous variable bipartite system. As for the theory, the qualitative characterization of the entanglement of twomode Gaussian states has been fully developed by determining the necessary and sufficient criteria for their separability [6, 7]. A computable quantitative characterization of entanglement for such states is available as well, being provided by negativity [8] and, in the symmetric instance, by the entanglement of formation [9 10].

Due to the interaction with the environment, any pure quantum state involved in some quantum information process evolves into a mixed state. Therefore, another property of crucial interest in Quantum Information Theory is quantifying the degree of mixedness of a quantum state. Let us briefly recall that the degree of mixedness of a quantum state $\varrho$ can be characterized either by the Von Neumann entropy $S_{V}(\varrho)$ or by the linear entropy $S_{L}(\varrho)$. Such quantities are defined as follows for continuous variable systems:

$$
\begin{aligned}
& S_{V}(\varrho) \equiv-\operatorname{Tr}(\varrho \ln \varrho) \\
& S_{L}(\varrho) \equiv 1-\operatorname{Tr}\left(\varrho^{2}\right) \equiv 1-\mu(\varrho)
\end{aligned}
$$

where $\mu \equiv \operatorname{Tr}\left(\varrho^{2}\right)$ denotes the purity of the state $\varrho$. The linear entropy of an arbitrary $n$-mode Gaussian state can be easily computed, whereas the evaluation of the Von Neumann entropy requires, in general, a more involved technical procedure. Making use of the Von Neumann entropy is however preferable, as it allows for a finer and more precise characterization of mixedness and correlations for multi-mode Gaussian states. In fact, the Von Neumann entropy is additive on tensor product states, unlike the linear entropy. Moreover, we will show that for two-mode Gaussian states the Von Neumann entropy depends on two symplectic invariants, while the linear entropy in completely determined by only one invariant. Finally, the knowledge of the Von Neumann entropy of a generic two-mode state allows to obtain the mutual information $I(\varrho) \equiv S_{V}\left(\varrho_{1}\right)+S_{V}\left(\varrho_{2}\right)-S_{V}(\varrho)$ (here $\varrho_{i}$ is the reduced density matrix of subsystem $i$ ), which quantifies the total amount of correlations (quantum plus classical) contained in a state [11].

The Von Neumann entropy of a $n$-mode Gaussian state has been determined in some remarkable works on the capacity of Gaussian channels by Holevo et al. [12]. In this letter we elucidate the mathematical structure introduced by these Authors, recasting it in a form that is conceptually simple and physically useful for applications and for 
explicit experimental schemes. To do so, we provide a thorough and detailed analysis of the basics of the symplectic framework restricting to the two-mode case, presenting a simple and transparent procedure to evaluate the Von Neumann entropy and the mutual information of twomode Gaussian states. Our approach is based on the explicit determination of the global symplectic invariants. The methodology we introduce naturally highlights the role played by the symplectic eigenvalues in characterizing the correlations encoded in multipartite systems.

\section{Two-mode Gaussian states}

Let us consider a two-mode continuous variable system, described by the Hilbert space $\mathcal{H}=\mathcal{H}_{1} \otimes \mathcal{H}_{2}$ resulting from the tensor product of the Fock spaces $\mathcal{H}_{k}$ 's. We will call $a_{k}$ the annihilation operator acting on the space $\mathcal{H}_{k}$. Likewise, $\hat{x}_{k}=\left(a_{k}+a_{k}^{\dagger}\right) / \sqrt{2}$ and $\hat{p}_{k}=-i\left(a_{k}-a_{k}^{\dagger}\right) / \sqrt{2}$ are the quadrature phase operators of the mode $k$, the corresponding phase space variables being $x_{k}$ and $p_{k}$. The set of Gaussian states is, by definition, the set of states with Gaussian characteristic functions and quasi-probability distributions. Therefore, a Gaussian state is completely characterized by its first and second statistical moments, that is, respectively, by the vector of mean values $\bar{X} \equiv\left(\left\langle\hat{x}_{1}\right\rangle,\left\langle\hat{p}_{1}\right\rangle,\left\langle\hat{x}_{2}\right\rangle,\left\langle\hat{p}_{2}\right\rangle\right)$ and by the covariance matrix $\sigma$

$$
\sigma_{i j} \equiv \frac{1}{2}\left\langle\hat{x}_{i} \hat{x}_{j}+\hat{x}_{j} \hat{x}_{i}\right\rangle-\left\langle\hat{x}_{i}\right\rangle\left\langle\hat{x}_{j}\right\rangle
$$

First moments will be unimportant to our aims, and we will set them to zero (as it is always possible by means of a local unitary transformation) without any loss of generality for our results. For simplicity, in what follows $\sigma$ will refer both to the Gaussian state and to its covariance matrix. It is convenient to express $\sigma$ in terms of the three $2 \times 2$ matrices $\boldsymbol{\alpha}, \boldsymbol{\beta}, \gamma$

$$
\boldsymbol{\sigma} \equiv\left(\begin{array}{cc}
\boldsymbol{\alpha} & \boldsymbol{\gamma} \\
\gamma^{T} & \boldsymbol{\beta}
\end{array}\right)
$$

Let us define two further submatrices of $\sigma$

$$
\boldsymbol{\delta}=\left(\begin{array}{ll}
\sigma_{11} & \sigma_{13} \\
\sigma_{31} & \sigma_{33}
\end{array}\right), \quad \boldsymbol{\epsilon}=\left(\begin{array}{ll}
\sigma_{22} & \sigma_{24} \\
\sigma_{42} & \sigma_{44}
\end{array}\right)
$$

The privileged role played by $\delta$ and $\epsilon$ in characterizing the action of global symplectic operations on $\sigma$ will become clear in the following.

Positivity of $\varrho$ and the commutation relations for quadrature phase operators impose the following constraint ensuring that $\sigma$ be a bona fide covariance matrix [6]

$$
\boldsymbol{\sigma}+\frac{i}{2} \boldsymbol{\Omega} \geq 0
$$

where $\Omega$ is the usual symplectic form

$$
\boldsymbol{\Omega} \equiv\left(\begin{array}{cc}
\boldsymbol{\omega} & 0 \\
0 & \boldsymbol{\omega}
\end{array}\right), \quad \boldsymbol{\omega} \equiv\left(\begin{array}{cc}
0 & 1 \\
-1 & 0
\end{array}\right) \text {. }
$$

Inequality 6 is a useful way to express the Heisenberg uncertainty principle.

In the following, we will make use of the Wigner quasiprobability representation $W\left(x_{i}, p_{i}\right)$, defined as the Fourier transform of the symmetrically ordered characteristic function [13]. In the Wigner phase space picture, the tensor product $\mathcal{H}=\mathcal{H}_{1} \otimes \mathcal{H}_{2}$ of the Hilbert spaces $H_{i}$ 's of the two modes results in the direct sum $\Gamma=\Gamma_{1} \oplus \Gamma_{2}$ of the related phase spaces $\Gamma_{i}$ 's. As a consequence of the Stone-Von Neumann theorem, a symplectic transformation acting on the global phase space $\Gamma$ corresponds to a unitary operator acting on $\mathcal{H}$ [14]. In what follows we will refer to a transformation $S_{l}=S_{1} \oplus S_{2}$, with each $S_{i} \in S p_{(2, \mathbb{R})}$ acting on $\Gamma_{i}$, as to a "local symplectic operation". The corresponding unitary transformation is the "local unitary transformation" $U_{l}=U_{1} \otimes U_{2}$, with each $U_{i}$ acting on $\mathcal{H}_{i}$. Inequality (6) is then a constraint on the $S p_{(2, \mathbb{R})} \oplus S p_{(2, \mathbb{R})}$ invariants [6]

$$
\operatorname{Det} \boldsymbol{\alpha}+\operatorname{Det} \boldsymbol{\beta}+2 \operatorname{Det} \boldsymbol{\gamma} \leq \frac{1}{4}+4 \operatorname{Det} \boldsymbol{\sigma}
$$

The Wigner function of a Gaussian state, written in terms of the phase space quadrature variables, reads

$$
W(X)=\frac{\mathrm{e}^{-\frac{1}{2} X \boldsymbol{\sigma}^{-1} X^{T}}}{\pi \sqrt{\operatorname{Det} \boldsymbol{\sigma}}},
$$

where $X$ stands for the vector $\left(x_{1}, p_{1}, x_{2}, p_{2}\right) \in \Gamma$. In general, the Wigner function transforms as a scalar under symplectic operations, while the covariance matrix $\sigma$ transforms according to

$$
\boldsymbol{\sigma} \rightarrow S^{T} \boldsymbol{\sigma} S, \quad S \in S p_{(4, \mathbb{R})} .
$$

As it is well known, for any covariance matrix $\boldsymbol{\sigma}$ there exists a local canonical operation $S_{l}=S_{1} \oplus S_{2}$ which brings $\boldsymbol{\sigma}$ in the "standard form" $\boldsymbol{\sigma}_{s f}[7]$

$$
S_{l}^{T} \boldsymbol{\sigma} S_{l}=\boldsymbol{\sigma}_{s f} \equiv\left(\begin{array}{cccc}
a & 0 & c_{1} & 0 \\
0 & a & 0 & c_{2} \\
c_{1} & 0 & b & 0 \\
0 & c_{2} & 0 & b
\end{array}\right)
$$

where $a, b, c_{1}, c_{2}$ are determined by the four local symplectic invariants Det $\boldsymbol{\sigma}=\left(a b-c_{1}^{2}\right)\left(a b-c_{2}^{2}\right)$, Det $\boldsymbol{\alpha}=a^{2}$, $\operatorname{Det} \boldsymbol{\beta}=b^{2}$, and Det $\boldsymbol{\gamma}=c_{1} c_{2}$. Therefore, the coefficients of the standard form corresponding to any covariance matrix are unique (up to a common sign flip of the $c_{i}$ 's). 


\section{Determination of the Von Neumann entropy}

To proceed, let us first note that the purity $\mu$ (and therefore the linear entropy $S_{L}$ ) of a Gaussian state can be easily computed. In fact, the trace of a product of operators corresponds to the integral of the product of their Wigner representations (when existing) over the whole phase space. Using the Wigner representation $W$ of $\varrho$ and taking into account the proper normalization factors, for a $n$-mode Gaussian state we get

$$
\mu=\frac{\pi}{2^{n}} \int_{\mathbb{R}^{2 n}} W^{2} \mathrm{~d}^{n} x \mathrm{~d}^{n} p=\frac{1}{2^{n} \sqrt{\operatorname{Det} \boldsymbol{\sigma}}} .
$$

Eq. (10) implies that a Gaussian state $\sigma$ is pure if and only if Det $\boldsymbol{\sigma}=1 / 2^{2 n}$.

For single-mode systems, the Von Neumann entropy can be easily computed as well. Neglecting first moments, any single-mode Gaussian state $\varrho$ can in fact be written as

$$
\varrho=S_{s m}(r, \varphi) \boldsymbol{\nu}_{\bar{n}} S_{s m}^{\dagger}(r, \varphi),
$$

where $\boldsymbol{\nu}_{\bar{n}}$ is a thermal state of mean photon number $\bar{n}$

$$
\boldsymbol{\nu}_{\bar{n}}=\frac{1}{1+\bar{n}} \sum_{k=0}^{\infty}\left(\frac{\bar{n}}{1+\bar{n}}\right)^{k}|k\rangle\langle k|,
$$

and $S_{s m}(r, \varphi)=\exp \left(\frac{1}{2} r \mathrm{e}^{-i 2 \varphi} a^{2}-\frac{1}{2} r \mathrm{e}^{i 2 \varphi} a^{\dagger 2}\right)$ is the single-mode squeezing operator. Being unitary, the latter does not affect the values of the traces in Eqns. (1)(2), computed on the diagonal density matrix $\nu_{\bar{n}}$ given by Eq. (12). One has then

$$
\begin{gathered}
\mu(\varrho)=\frac{1}{2 \bar{n}+1}=\frac{1}{2 \sqrt{\operatorname{Det} \boldsymbol{\sigma}}} \\
S_{V}(\varrho)=\bar{n} \ln \left(\frac{\bar{n}+1}{\bar{n}}\right)+\ln (\bar{n}+1) \\
=\frac{1-\mu}{2 \mu} \ln \left(\frac{1+\mu}{1-\mu}\right)-\ln \left(\frac{2 \mu}{1+\mu}\right) .
\end{gathered}
$$

Eq. [14], first achieved in Ref. [15], shows that for singlemode Gaussian states the Von Neumann entropy is a monotonically increasing function of the linear entropy, so that $S_{V}$ and $S_{L}$ yield the same characterization of mixedness. In fact, both $S_{V}$ and $S_{L}$ are fully determined by the same symplectic invariant Det $\sigma$. As we will now see, this is no longer true for two-mode Gaussian states.

To find an expression for the Von Neumann entropy of a generic Gaussian state of a two-mode system, we must find a general expression for the state analogous to that provided by Eq. 111 for a single-mode system. Neglecting first moments, this amounts to determine the most general parametrization of the covariance matrix, which is provided by the following lemma.

Lemma 1 An arbitrary two-mode covariance matrix $\boldsymbol{\sigma}$ can be written as

$$
\boldsymbol{\sigma}=A^{T} \boldsymbol{\nu}_{n_{\mp}} A,
$$

where $\boldsymbol{\nu}_{n_{\mp}}=\boldsymbol{\nu}_{n_{-}-1 / 2} \oplus \boldsymbol{\nu}_{n_{+}-1 / 2}$ is the covariance matrix of a tensor product of single-mode thermal states with average photon number $\bar{n}_{\mp} \equiv n_{\mp}-1 / 2$ in the two modes

$$
\boldsymbol{\nu}_{n_{\mp}}=\operatorname{diag}\left(n_{-}, n_{-}, n_{+}, n_{+}\right) \text {, }
$$

while

$$
A=S_{l o c}\left(r_{1}, r_{2}\right) R(\xi) S_{t m}(r) R(\eta) S_{l}
$$

is a symplectic operation belonging to $S p_{(4, \mathbb{R})}$. Transformation $A$ is made up by a local operation $S_{l}$, two rotations $R(\phi)$, with

$$
R(\phi)=\left(\begin{array}{cccc}
\cos \phi & 0 & -\sin \phi & 0 \\
0 & \cos \phi & 0 & -\sin \phi \\
\sin \phi & 0 & \cos \phi & 0 \\
0 & \sin \phi & 0 & \cos \phi
\end{array}\right),
$$

a two-mode squeezing $S_{t m}(r)=\operatorname{diag}\left(\mathrm{e}^{r}, \mathrm{e}^{-r}, \mathrm{e}^{-r}, \mathrm{e}^{r}\right)$ and a local squeezing $S_{l o c}\left(r_{1}, r_{2}\right)=S_{s m}\left(r_{1}, 0\right) \oplus$ $S_{s m}\left(r_{2}, 0\right)$, resulting from the direct product of two singlemode squeezing operators with null phase. Note that $S_{t m}(r)=S_{l o c}(r,-r)$, so that the only global (nonlocal) operations in the decomposition of Eq. 177 are the two rotations. We note that an equivalent decomposition has been recently demonstrated for generic multimode pure Gaussian states [16]: the authors have shown that for any decomposition of a multimode pure Gaussian state with respect to a bipartite division of the modes, the state can always be expressed as a product state involving entangled two-mode squeezed states and single-mode local states at each side.

Proof. In order to prove the statement expressed by Eq. 15, we consider the equivalent expression $A^{-1 T} \boldsymbol{\sigma} A^{-1}=\boldsymbol{\nu}_{n_{\mp}}$ and show that it is realized by some $A, n_{-}, n_{+}$for any given $\sigma$.

First, we choose $S_{l}$ to bring $\sigma$ to its standard form, given by Eq. (9). We then apply to $\sigma_{s f}$ the rotated two-mode squeezing $R(\eta)^{-1} S_{t m}(r)^{-1}$, taking the covariance matrix to the form

$$
\left(\begin{array}{cccc}
s & 0 & 0 & 0 \\
0 & m & 0 & c \\
0 & 0 & s & 0 \\
0 & c & 0 & n
\end{array}\right)
$$

which is convenient, due to the invariance of the submatrix $\boldsymbol{\delta}=\operatorname{diag}(s, s)$, see Eqns. [5], under two-mode rotations of the form Eq. 18. The second rotation $R(\xi)^{-1}$ leaves 
$\delta$ unchanged and can be chosen to make $c$ null, yielding a state of the form

$$
\left(\begin{array}{cccc}
s & 0 & 0 & 0 \\
0 & m^{\prime} & 0 & 0 \\
0 & 0 & s & 0 \\
0 & 0 & 0 & n^{\prime}
\end{array}\right),
$$

which can be finally put in the desired form $\boldsymbol{\nu}_{n_{-}-1 / 2} \oplus$ $\nu_{n_{+}-1 / 2}$ by means of the local squeezing $S_{l o c}\left(r_{1}, r_{2}\right)$.

Lemma 1 introduces an equivalence relation on the set of Gaussian states, associating to any Gaussian state $\sigma$ a product of thermal states $\boldsymbol{\nu}_{n_{\mp}}$, by means of the correspondence defined by Eq. (15). The quantities $n_{\mp}$ are known as the symplectic eigenvalues of $\boldsymbol{\sigma}$, while transformation $A$ performs a symplectic diagonalization [12,8 17]. We note that the decomposition of the symplectic operation $A$ given by Eq. 177 is the particular two-mode case of the general decomposition of a symplectic operation [18].

Let us now focus on the quantity

$$
\Delta(\boldsymbol{\sigma})=\operatorname{Det} \boldsymbol{\alpha}+\operatorname{Det} \boldsymbol{\beta}+2 \operatorname{Det} \boldsymbol{\gamma},
$$

where $\boldsymbol{\alpha}, \boldsymbol{\beta}$, and $\boldsymbol{\gamma}$ are defined as in Eq. (4). We have:

Lemma 2. $\Delta(\boldsymbol{\sigma})$ is invariant under the action of the symplectic transformation A defined by Eq. (15).

Proof. $\Delta(\sigma)$ is clearly invariant under local operations, such as $S_{l}, S_{l o c}$ and $S_{t m}$. As for the non local rotations which enter in the definition of $A$, let us notice that they act on covariance matrices of the following form

$$
\tilde{\boldsymbol{\sigma}}=\left(\begin{array}{cccc}
u & 0 & j & 0 \\
0 & v & 0 & k \\
j & 0 & w & 0 \\
0 & k & 0 & z
\end{array}\right),
$$

for which one has

$$
\Delta(\tilde{\boldsymbol{\sigma}})=\operatorname{Tr}(\boldsymbol{\delta} \boldsymbol{\epsilon})
$$

Such an expression is manifestly invariant under the action of identical rotations $R(\phi)$ on the submatrices $\delta$ and $\gamma$, see Eqns. (5) and (18).

The quantity Det $\boldsymbol{\sigma}$ is obviously invariant as well under the action of $A$ since, for any symplectic transformation $S$, one has Det $S=1$. Exploiting the invariance of Det $\sigma$ and $\Delta(\boldsymbol{\sigma})$ one can determine the symplectic eigenvalues $n_{\mp}$ which characterize a generic Gaussian state $\sigma$, according to Eq. 15

$$
\begin{aligned}
& \operatorname{Det} \boldsymbol{\sigma}=\operatorname{Det} \boldsymbol{\nu}_{n_{\mp}}=n_{-}^{2} n_{+}^{2}, \\
& \Delta(\boldsymbol{\sigma})=\Delta\left(\boldsymbol{\nu}_{n_{\mp}}\right)=n_{-}^{2}+n_{+}^{2} .
\end{aligned}
$$

The solution of the system yields

$$
n_{\mp}(\boldsymbol{\sigma})=\sqrt{\frac{\Delta(\boldsymbol{\sigma}) \mp \sqrt{\Delta(\boldsymbol{\sigma})^{2}-4 \operatorname{Det} \boldsymbol{\sigma}}}{2}} .
$$

Note that Ineq. (6) is equivalent to

$$
n_{\mp} \geq \frac{1}{2},
$$

whereas the necessary and sufficient criterion for a state to be pure reads $n_{-}=n_{+}=1 / 2$ (one can easily show that it is equivalent to Det $\boldsymbol{\sigma}=1 / 16$ ).

Knowledge of the symplectic eigenvalues and of the associated mean thermal photon numbers allows finally to determine the Von Neumann entropy $S_{V}(\boldsymbol{\sigma})$ of an arbitrary two-mode Gaussian state $\sigma$. We have:

Proposition 1. The Von Neumann entropy $S_{V}(\boldsymbol{\sigma})$ of an arbitrary two-mode Gaussian state $\sigma$ equals the one of the tensor product of thermal states $\nu_{n_{\mp}}$, associated to $\boldsymbol{\sigma}$ via the correspondence established by Eq. (15), and its expression reads

$$
S_{V}(\boldsymbol{\sigma})=f\left[n_{-}(\boldsymbol{\sigma})\right]+f\left[n_{+}(\boldsymbol{\sigma})\right],
$$

with $n_{\mp}$ given by Eqns. 21) and

$$
f(x) \equiv\left(x+\frac{1}{2}\right) \ln \left(x+\frac{1}{2}\right)-\left(x-\frac{1}{2}\right) \ln \left(x-\frac{1}{2}\right) .
$$

Proof. The symplectic operation $A$ described by Eq. (17) corresponds to a unitary transformation in the Hilbert space $\mathcal{H}$ which cannot affect the value of the trace appearing in the definition of $S_{V}$, according to Eq. (1). Therefore, exploiting Eq. 14 and the additivity of the Von Neumann entropy for tensor product states, one obtains Eq. 23.

We have shown that the Von Neumann entropy of a twomode Gaussian state $\sigma$ depends on the two invariants $\Delta(\sigma)$ and Det $\boldsymbol{\sigma}$, whereas the purity of $\boldsymbol{\sigma}$ is completely determined by Det $\boldsymbol{\sigma}$ alone, just as in the single-mode case. This implies that the hierarchy of mixedness established by the Von Neumann entropy on the set of Gaussian states differs, in the two-mode case, from that induced by the linear entropy. States may exist with a given linear entropy, i.e. with a given Det $\sigma$, but with different Von Neumann entropies, i.e. with different $\Delta(\sigma)$ 's. The Von Neumann entropy thus provides a richer characterization of the state's lack of information.

\section{Symplectic eigenvalues, mutual infor- mation and correlations}

The mutual information $I(\boldsymbol{\sigma})$ of a Gaussian state $\boldsymbol{\sigma}$ is defined as

$$
I(\boldsymbol{\sigma})=S_{V}\left(\boldsymbol{\sigma}_{1}\right)+S_{V}\left(\boldsymbol{\sigma}_{2}\right)-S_{V}(\boldsymbol{\sigma}),
$$


where $\sigma_{i}$ stands for the reduced single-mode state obtained by tracing over subsystem $j \neq i$. Knowledge of $S_{V}(\boldsymbol{\sigma})$ leads to the following:

Proposition 2. The mutual information $I(\sigma)$ of an arbitrary two-mode Gaussian state is

$$
I(\boldsymbol{\sigma})=f(a)+f(b)-f\left[n_{-}(\boldsymbol{\sigma})\right]-f\left[n_{+}(\boldsymbol{\sigma})\right],
$$

where $a=\sqrt{\operatorname{Det} \boldsymbol{\alpha}}, b=\sqrt{\operatorname{Det} \boldsymbol{\beta}}$, and $f(x)$ is the same as in Eq. 24.

Proof. Let us consider the reduction of $\sigma$ to its standard form $\sigma_{s f}$, defined by Eq. (9). The matrix elements $a$ and $b$ of $\sigma_{s f}$ are easily recovered from a generic $\sigma$, because $\operatorname{Det} \boldsymbol{\alpha}=a^{2}$ and $\operatorname{Det} \boldsymbol{\beta}=b^{2}$ are $S p_{(2, \mathbb{R})} \oplus S p_{(2, \mathbb{R})}$ invariant. Notice that, since either $S_{V}(\boldsymbol{\sigma})$ or the quantities $S_{V}\left(\boldsymbol{\sigma}_{i}\right)$ 's are invariant under local unitary operations, one has $I(\boldsymbol{\sigma})=I\left(\boldsymbol{\sigma}_{s f}\right)$. Partial tracing of $\boldsymbol{\sigma}_{s f}$ over subsystem $i$ yields $\boldsymbol{\sigma}_{1}=\operatorname{diag}(a, a)$ and $\boldsymbol{\sigma}_{2}=\operatorname{diag}(b, b)$, so that, finally, Eq. (14) and Proposition 1 lead to Eq. (26).

Notice that $a$ and $b$ constitute the symplectic spectra of, respectively, $\sigma_{1}$ and $\sigma_{2}$. Eq. (26) emphasizes the relevant role played by the symplectic eigenvalues $n_{\mp}\left(\sigma_{s f}\right)$ in determining the total amount of correlations contained in a quantum state of a continuous variable system, in striking analogy to the role played by the symplectic eigenvalues of the partial transpose of $\sigma_{s f}$ in characterizing the amount of quantum correlations [9, 10].

To better clarify this point, let us consider a symmetric state $\sigma_{\text {sym }}$, i.e. a state whose standard form fulfills $a=b$, so that its mutual information reads, according to Eq. (26)

$$
I\left(\boldsymbol{\sigma}_{\text {sym }}\right)=2 f(a)-f\left[n_{-}\left(\boldsymbol{\sigma}_{\text {sym }}\right)\right]-f\left[n_{+}\left(\boldsymbol{\sigma}_{\text {sym }}\right)\right],
$$

with symplectic eigenvalues $n_{\mp}=\sqrt{\left(a \mp c_{1}\right)\left(a \mp c_{2}\right)}$. On the other hand, the symplectic eigenvalues of the partially transposed covariance matrix $\tilde{\boldsymbol{\sigma}}_{\text {sym }}$ (obtained from $\sigma_{\text {sym }}$ by switching the sign of $c_{2}$, see [6]) are $\tilde{n}_{\mp} \equiv n_{\mp}\left(\tilde{\boldsymbol{\sigma}}_{\text {sym }}\right)=\sqrt{\left(a \mp c_{1}\right)\left(a \pm c_{2}\right)}$. In particular, for an entangled state, the smallest eigenvalue is $\tilde{n}_{-}=$ $\sqrt{\left(a-\left|c_{1}\right|\right)\left(a-\left|c_{2}\right|\right)}$.

The symplectic eigenvalue $\tilde{n}_{-}$encodes all the information about the entanglement of the state, since the necessary and sufficient criterion for entanglement reduces to $\tilde{n}_{-}<1 / 2$, while the entanglement of formation $E_{F}\left(\boldsymbol{\sigma}_{\text {sym }}\right)$ reads ${ }^{2}$

$$
E_{F}\left(\boldsymbol{\sigma}_{\text {sym }}\right)=\max \left\{0, g\left[\tilde{n}_{-}\right]\right\},
$$

with $g(x) \equiv \frac{\left(\frac{1}{2}+x\right)^{2}}{2 x} \ln \left(\frac{\left(\frac{1}{2}+x\right)^{2}}{2 x}\right)-\frac{\left(\frac{1}{2}-x\right)^{2}}{2 x} \ln \left(\frac{\left(\frac{1}{2}-x\right)^{2}}{2 x}\right)$ (see [9] for details); it correctly reduces to $I(\boldsymbol{\sigma}) / 2$ for pure

\footnotetext{
${ }^{1}$ If $\boldsymbol{\sigma}$ is entangled, then Det $\boldsymbol{\gamma}<0$, see Ref. [6].

${ }^{2} E_{F}(\varrho) \equiv \min _{\left\{p_{i},\left|\psi_{i}\right\rangle\right\}} \sum p_{i} E\left(\left|\psi_{i}\right\rangle\left\langle\psi_{i}\right|\right)$, where $E(|\psi\rangle\langle\psi|)$ is the entropy of entanglement of the pure state $|\psi\rangle$, defined as the Von Neumann entropy of its reduced density matrix, and the min is taken over all the pure states realization of $\varrho=\sum p_{i}\left|\psi_{i}\right\rangle\left\langle\psi_{i}\right|$.
}

symmetric states. Even the quantification of quantum correlation provided by negativity [8], which is computable also for non symmetric states, reduces for a two-mode Gaussian state $\boldsymbol{\sigma}$ to a simple function of $\tilde{n}_{-}(\boldsymbol{\sigma})$.

The dependence of entanglement on the eigenvalue $\tilde{n}_{-}(\boldsymbol{\sigma})$ is due to the fact that the biggest eigenvalue of the partially transposed covariance matrix $\tilde{\sigma}$ can be easily shown to fulfill Ineq. 22). Thus $\tilde{n}_{-}(\boldsymbol{\sigma})$ alone can be responsible of the violation of the PPT ('positivity of the partial transpose') criterion for separability [6], which can be recast as

$$
\tilde{n}_{-}(\boldsymbol{\sigma}) \geq \frac{1}{2} .
$$

All the quantification of entanglement for two-mode Gaussian states available at present just quantify the violation of this inequality.

We have extensively shown how both quantum and classical correlations of a Gaussian state are encoded in symplectic spectra of the global, reduced and partially transposed covariance matrices of the state.

\section{Conclusions}

In conclusion, we have characterized mixedness and total correlations of two-mode Gaussian states by deriving their Von Neumann entropy and mutual information. Comparing these quantities with the entanglement of formation of symmetric states shows that a crucial information about quantum and classical correlations lies in the symplectic eigenvalues of the covariance matrix and of its partial transpose. The problem is still left open of determining a fully satisfactory quantification of the purely quantum correlations in a general two-mode Gaussian state.

\section{Acknowledgements}

Financial support from INFM, INFN, and MIUR under national project PRIN-COFIN 2002 is acknowledged.

\section{References}

[1] Quantum Information Theory with Continuous Variables, S. L. Braunstein and A. K. Pati Eds. (Kluwer, Dordrecht, 2002).

[2] H. J. Kimble and D. F. Walls, J. Opt. Soc. Am. B 4, 10 (1987).

[3] H. P. Yuen and A. Kim, Phys. Lett. A 241, 135 (1998); F. Grosshans, G. Van Assche, J. Wenger, R. Brouri, N. J. Cerf, and P. Grangier, Nature 421, 238 (2003). 
[4] A. Furusawa, J. L. Sorensen, S. L. Braunstein, C. A. Fuchs, H. J. Kimble, and E. S. Polzik, Science 282, 706 (1998); T. C. Zhang, K. W. Goh, C. W. Chou, P. Lodahl, and H. J. Kimble, Phys. Rev. A 67, 033802 (2003).

[5] S. Lloyd and S. L. Braunstein, Phys. Rev. Lett. 82, 1784 (1999); T. C. Ralph, W. J. Munro, and G. J. Milburn, quant-ph/0110115 (2001).

[6] R. Simon, Phys. Rev. Lett. 84, 2726 (2000).

[7] L.-M. Duan, G. Giedke, J. I. Cirac, and P. Zoller, Phys. Rev. Lett. 84, 2722 (2000).

[8] G. Vidal and R. F. Werner, Phys. Rev. A 65, 032314 (2001).

[9] G. Giedke, M. M. Wolf, O. Krüger, R. F. Werner, and J. I. Cirac, Phys. Rev. Lett. 91107901 (2003).

[10] M. M. Wolf, G. Giedke, O. Krüger, R. F. Werner, and J. I. Cirac, quant-ph/0306177 (2003).

[11] L. Henderson and V. Vedral, J. Phys. A 34, 6899 (2001).

[12] A. S. Holevo, M. Sohma, and O. Hirota, Phys. Rev. A 59, 1820 (1999); A. S. Holevo and R. F. Werner, ibid. 63, 032312 (2001).

[13] See, e.g., S. M. Barnett and P. M. Radmore, Methods in Theoretical Quantum Optics (Clarendon Press, Oxford, 1997).

[14] R. Simon, E. C. G. Sudarshan, and N. Mukunda, Phys. Rev. A 36, 3868 (1987).

[15] G. S. Agarwal, Phys. Rev. A 3, 828 (1971).

[16] A. Botero and B. Reznik, Phys. Rev. A 67, 052311 (2003).

[17] J. Williamson, Am. J. Math. 58, 141 (1936).

[18] H. Huang and G. S. Agarwal, Phys. Rev. A 49, 52 (1994). 